\title{
Expert vs. Peer: The Role of Situational Characteristics in Electronic Networks of Practice
}

\author{
Matthew Jensen \\ University of Oklahoma \\ mjensen@ou.edu
}

\author{
Michael Matthews \\ University of Oklahoma \\ michael.j.matthews- \\ 1@ou.edu
}

\author{
Thomas Meservy \\ Brigham Young \\ University \\ tmeservy@byu.edu
}

\author{
Kelly Fadel \\ Utah State University \\ kelly.fadel@usu.edu
}

\begin{abstract}
Individuals turn to online forums sponsored by Electronic Networks of Practice (ENPS) to acquire information on a range of topics; yet, quality and relevancy of such information can vary greatly. To assist information seekers, many ENP forums incorporate contextual cues that provide signals originating from both expert and lay forum users about the quality of solutions. While extant research has explored how these cue sources influence information filtering on ENP forums, results on their relative impact are fragmented and often contradictory. This paper advances research in this domain by employing situation theory to examine how six situational characteristics influence individuals' propensity to rely on peer vs. expert recommendations. Results demonstrate that users rely more on expert recommendations when seeking information for situations that they perceive as stressful, task-oriented, or requiring greater cognitive processing, while peer recommendations are preferred for situations perceived as fun. Implications for research and practice are discussed.
\end{abstract}

\section{Introduction}

"How do we know who to believe in an [online environment] where anyone can say anything about anything to everyone" [1, p. 673]; emphasis original.

The dawn of Web 2.0 represented new era of online information exchange wherein lay users who were previously confined to consuming content could suddenly produce content as well [2]. Among the new information exchange structures born from this shift are Electronic Networks of Practice (ENPs), defined as "computer-mediated social spaces where individuals working on similar problems self-organize to help each other and share knowledge, advice, and perspectives about their occupational practice or common interests" [3, p. 254]. Information exchange in ENPs can take many forms but occurs most commonly via online discussion forums, technology-mediated online question/answer platforms typically devoted to discussion about a specific topic or domain (e.g., software programming, health, tax code, law, fishing). Using an online forum, ENP participants can post their own questions, receive and review answers from other participants, and search previous question/answer threads initiated by other participants. Attesting to their popularity as a platform for information exchange, a popular press survey found that 72 percent of respondents indicated that online forums are reliable for trustworthy information and 88 percent belonged to at least one online forum [4].

The open and loosely regulated nature of most ENP forums means that answers posted in response to forum questions can originate from wide variety of participants and may therefore exhibit significant variation in quality. This places a cognitive burden on forum users who must often evaluate and filter several competing candidate solutions to a given question. To aide in this filtering process, ENP forums usually offer contextual cues that provide signals from other sources as to the purported quality of each solution. For example, many forums provide visual cues that indicate whether a solution was accepted by the original question poster, endorsed by a subject matter expert, or verified by other members of the community.

One of the primary features that distinguishes ENP forum contextual cues from each other is their source; namely, the nature and characteristics of the person(s) who provides the feedback indicated by the cue. Some cues reflect the opinion of expert forum users, i.e., individuals that have been credentialed or certified to have advanced knowledge in a given subject area. For instance, online forums such as doctorslounge.com indicates profession - and thereby a level of expertise - directly below the respondents' avatar (e.g., pharmacist, nurse, medical doctor, guest). 
Complementing expert cues, most forums also feature cues that originate from "regular" (peer) forum users with no particular expertise or credentials. For example, the website forums such as patient.info, inspire.com and medhelp.org allow individuals without any credentialed medical expertise to provide advice and suggestions to mental and physical health peer-generated questions. Emerging research on ENP forums suggests a role for both expert- and peer-based cues in shaping information filtering decisions [5]-[8]; yet, these studies also exhibit discrepancies as to the relative influential magnitude of these cue sources. More empirical and theoretical work is needed to elucidate how, to what degree, and under what circumstances different sources of contextual cues influence information filtering decisions.

This paper reports the results of an exploratory study designed to better understand the circumstances under which people rely on opinions of experts vs. those of their peers when evaluating information for problem solving, such as that typically found on an ENP forum. The central thesis of our study is that reliance on peervs. expert-based cues on an ENP forum depends, in part, on specific situational factors that characterize the nature of the question and the knowledge required to answer it. We draw from literature in situational psychology to identify six situational characteristics that describe different types of problems/questions for which one might search for solutions on an ENP forum. Then, using literature from consumer product and service domains, we develop hypotheses about how each characteristic is likely to shape reliance on peer vs. expert opinions. We test our hypotheses using data from an online survey that asked participants to rate situational characteristics for each of 20 scenarios, as well as their predisposition to seek expert vs. peer advice for each scenario. The results of this study provide an important theoretical groundwork for a broader research program aimed at better understanding how different contextual cue attributes influence information filtering on ENP forums.

\section{Theoretical Background}

"It is axiomatic that when one seeks information about almost any topic with as many potential participants as one finds on the Internet, one is confronted with a myriad of opinions. A corollary of this axiom is that, if one wishes to make a judgment about a topic about which one has sought information, one must evaluate multiple_often highly discrepant-sources." Van Der Heide et al. [1, p. 674]

Contextual cues on an ENP forum are visual indicators, usually in graphical or symbolic form, designed to inform users of the likely quality of an object, person, or idea that is presented on the forum [9]. Contextual cues are commonly attached to forum solutions - i.e., answers to questions of knowledgeseeking forum users that are supplied by knowledgeproviding forum users. Contextual cues associated with solutions vary widely in their type, format, and source. For example, many forums offer a binary cue (such as a check mark) that signals whether a particular solution has been accepted by the question poster. In addition, some forums feature cues that reflect the endorsement or rebuttal of subject matter experts who are credentialed by forum moderators or established by community consensus. Yet other cues reflect the feedback of the wider community of peer forum users. For example, forums frequently allow any user to rate solutions with up- and down-votes, claps, or ordinal (e.g., star) rating scales. Each of these types of cues can provide a unique signal as to the underlying quality of an ENP forum solution. However, most forums feature not just one type of contextual cue, but some combination of cues originating from the sources described above. Information seekers, therefore, often encounter unique cue combinations as they evaluate competing candidate solutions to a given problem. For instance, one solution may be endorsed by the original poster but refuted by an expert user. Another solution may be ratified by an expert but lack endorsement from the larger community of users. Knowledge seekers must therefore weigh signals from different cue sources as they filter candidate solutions en route to a final adoption decision.

Recent research has begun to elucidate the important role of contextual cues in the ENP information filtering process, showing that these cues influence even experienced knowledge seekers with the capacity to evaluate solutions on their own merits [10]. Although not a central focus of this research stream todate, results from this body of work have also suggested that certain contextual cue sources may be more influential than others; nevertheless, initial results have been somewhat inconsistent. Meservy et al. [8], for example, conducted an experiment in which experienced software developers were asked to evaluate solutions to common programming problems found on ENP forums. Their results indicated that cues signaling the feedback of community members weighed more heavily in user's decisions than cues signaling the approval/disapproval of a forum expert. Such findings are corroborative of other studies that have also observed the superior influence of community cues [7]. However, research in other domains suggests that expert opinions may be preferred over those of other community members in some cases [11]. Thus far, there is a lack of theoretical and empirical work to explain these discrepant results. 
One potential explanation for the divergent outcomes of previous work is that reliance on peer vs. expert-based cues depends in part on key characteristics of the situation in question. Defined generally, a situation is a set of circumstances or state of affairs in which one finds oneself [12]. For the purposes of this paper, we use "situation" to refer to the nature of the circumstances surrounding a question posed on an ENP forum and the corresponding information needed to address it. For example, repairing a vehicle, solving a math problem, finding a romantic mate, or deciding on an elective surgery could all represent situations for which one might search for answers on an ENP forum. Each situation possesses unique attributes that differentiate it from other situations. Some situations, for example, could be related to the attainment of a task, require complex solutions, or entail a high degree of risk, while other situations may involve lower stakes decisions related to hedonic or preferential pursuits.

The study of situations and their effect on human behavior has a substantial and growing presence in social psychology research [13], [14]. A primary aim of this literature is to delineate the nature of situations and their distinguishing characteristics in order to build theory that can predict and explain human behavior from a situational perspective. To frame such theory, theorists distinguish between the objective physical cues that define a situation (i.e., measurable factors that identify who, what, where, when, etc.) and the subjective characteristics that describe how the situation is psychologically interpreted and imbued with meaning by those who experience it [15]. Of course, two individuals who experience the same objective situational cues (e.g., being set up on a blind date or challenged to a fight) may react very differently (e.g., responding with enthusiasm or beating a hasty retreat); thus, for the purposes of understanding how situations shape behavior, recent work has emphasized subjective characteristics that capture the psychologically important meaning of perceived situational cues.

One of the major undertakings of situational psychology research is identifying a tractable set of characteristics that capture an array of situational interpretations and experiences. In recent years, scholars have employed both lexical and theory-based methods to develop independent taxonomies of situational characteristics (e.g., [16]-[19]) each of which captures a different level of abstraction and is designed for a specific situational domain [14]. Synthesizing this literature and observing the conceptual similarities across taxonomies, Horstmann et al. [20] distilled approximately 30 taxonomic dimensions into six core situational characteristics that appear repeatedly across independent research efforts: Threat, Stress, Tasks, Processing, Fun, and Mundane.
These summative characteristics, which we employ in our research hypotheses, are described further in the following paragraph.

Threat describes situations where there is perceived risk or danger, either physical or psychological, that emanates from an external source. The threat may or may not be perceived negatively, depending on the individual's ability to cope with the threatening force. In a similar vein, stress refers to situations that are perceived as stressful or tense; however, stressful situations are distinguished from threatening situations (which are potentially but not necessarily stressful) in that "situations characterized by high stress tend to be more internally focused (e.g., situation is potentially anxiety inducing) than those characterized by threat, which typically stem from something external" [20, p. 15]. Tasks describes situations that involve following a specific set of steps to arrive at the completion of a job, attainment of an objective, or the fulfilling of a duty. Task-oriented situations often involve close attention to detail to avoid costly mistakes. Processing refers to situations that are subjectively complex and require thinking, analysis, and intellectual engagement. In contrast, fun describes situations that are social, positive, enjoyable, or otherwise hedonic in nature. Finally, mundane characterizes situations that are perceived as routine, normal, boring, or lacking in stimuli.

Horstmann et al.'s condensed taxonomy of psychological situation characteristics provides a useful theoretical framework for examining how different types of human behaviors are affected by situational influences. Using this framework, we now turn our attention to the focal behavior in the present study, namely, the extent to which people rely on the opinion or experts vs. peers when evaluating solutions on an ENP forum. To our knowledge, no study has explored how ENP information filtering is influenced by situational factors; thus, no theory is available to directly inform our hypotheses. Nevertheless, the influence of peer and expert opinions has received some attention in the consumer product and service literature, which offers useful insights for our current inquiry. In the following section, we draw on findings from this literature to develop our research hypotheses.

\section{Hypotheses}

We first consider circumstances under which people are more likely to rely on advice of an expert over that of a peer. Following prior research, we define an expert as someone possessing a high degree of domain expertise, based upon which s/he can offer a recommendation that is more professional, authoritative, or formal in nature [21]. Expert reviews 
offer value in that they are based on a depth of understanding or knowledge that exceeds that possessed by the consumer. Thus, expert endorsements can serve to reduce uncertainty in situations where consumers lack the knowledge, skill, or ability to evaluate information on its own merits. Such a scenario is likely in situations that involving a high degree of risk or uncertainty, which are more likely to be associated with perceptions of threat or stress. Research indicates that decisions that involving high uncertainty warrant information from more knowledgeable and credible sources to mitigate the uncertainty [22]. For example, consumers considering a highly risky purchase tend to seek additional information from others who are perceived to have a high level of expertise and knowledge [23], and the influence of expert comments is greater in a risky context [24]. Similarly, research suggests that people are more likely to rely on expert opinions in situations that are highly complex or that required a greater degree of cognitive processing than the consumer can achieve on her own [25]. Results from research in the medical domain, for example, suggests that individuals rely on experts to make sense of scientific knowledge in medicine [26] or to make judgments about the technical quality of procedures and equipment [27]. Finally, research suggests that reliance on expert recommendations may be greater in utilitarian scenarios involving a task to be accomplished, as opposed to hedonic or preferential situations. For example, Smith et al. [28] found that when subjects were asked to select a restaurant for a utilitarian purpose (e.g., a business meeting), recommender expertise had had a much stronger effect on the perceived influence of the recommendation than when they completed the same task for a hedonic purpose. Moreover, shoppers relied more on an expert reviewer's opinion nearly twice as much as on a peer's opinion when the task was utilitarian rather than hedonic in nature. Based on findings from this literature, we hypothesize the following:

H1: ENP forum users rely more on expert-based cues in situations perceived to be higher in threat, stress, processing, or task characteristics.

We next consider the situational factors that would prompt greater reliance on peer recommendations. In this context, "peer" refers to another user of an ENP forum with no particular credentialed expertise [21]. Social influence theory suggests that the opinions of others can exert influence on beliefs and behavior through both normative (i.e., desire to conform) and informational (i.e., internal adoption of others' beliefs) channels [29] When people encounter signals from peers about a solution on an ENP forum, they may be induced to adjust their beliefs about the solution to accord with these signals [30]. Research suggests that this adjustment is particularly likely when the person identifies closely or believes to share rapport with the peer recommender. However, evidence also suggests that the effect of peer opinions vis-à-vis those of experts may be stronger in certain situations than in others. For example Keh et al. [21] found that consumers relied more heavily on peer opinions vs. those of experts when tasked with rating more hedonic "experience" services such as movies or food, while expert reviews were more salient for "credence" services such as tax accounting, medical care, or financial planning. Even when holding the service constant (e.g., selecting a restaurant), research suggests that reliance on peer opinions is more likely when it is evaluated for a fun or hedonic purpose rather than a utilitarian purpose [28]. Moreover, complementing studies that show a greater reliance on expert opinions for situations involving a high degree of complexity or cognitive processing, there is also evidence to suggest that peer evaluations may be more influential in more mundane situations that require less cognitive effort, such as everyday preferential choice tasks with relatively minor consequences (i.e., purchasing a book) [5]. We therefore hypothesize a stronger influence of peer-based cues in situations that are perceived to be fun or mundane.

H2: ENP forum users rely more on peer-based cues in situations perceived to be higher in fun or mundane characteristics.

\section{Research Method}

To test our hypotheses, we designed a survey instrument that presented participants with twenty hypothetical situations (see Appendix A) taken from a popular press article [31]. These situations were chosen as likely to exhibit variation in reliance on peer vs expert opinions [31] and because they represented topics for which one might seek answers on an ENP forum. The survey included two types of questions used to test the hypotheses. First, participants were given a definition of each of the six situational characteristics and asked to rate the degree to which each characteristic described each situation. (Items were measured on a 1-5 ordinal scale, with 5 indicating agreement that the situation exhibited that situational factor and 1 indicating disagreement.) Second, for each situation, participants were asked to what extent they would (a) rely on an expert recommendation and (b) rely on a peer recommendation. To avoid confounds attributable to fatigue or ordering effects, the survey instrument 
randomized the presentation order of both situations and situational characteristics.

We recruited participants $(n=119)$ from Amazon's Mechanical Turk workforce to complete the study. Recruited participants were from the United States, had completed more than 100 HITs with a $95 \%$ or greater satisfaction rate, and held MTurk Master status. Participants were compensated \$1.50 USD for successfully completing the study.

\subsection{Data Analysis}

To analyze our survey data, we employed the lmer function of the lme 4 package in $\mathrm{R}$ [32] to estimate a series of mixed effects regression models to examine which situational characteristics (IVs) were associated with reliance on peer vs. expert recommendations (DVs). Mixed effects models were used because the data we collected were hierarchal in nature: each participant rated each situation multiple times - once for each of the dimensions. To assess the presence of random effects attributable to the participant and situation, we first compared a baseline (intercept-only) model with a random-effects-only model for each DV using participant and situation as random factors. Comparison of model fit parameters indicated that the fit of the random-effects-only models was significantly higher than the baseline for both expert (Table 1; $\chi 2=$ 1472.7, $\mathrm{p}<.001$ ) and peer (Table $3 ; \chi 2=909.8, \mathrm{p}<$ $.001)$, suggesting that these random effects accounted for some variance in the dependent variables. To gauge the relative impact of these random factors, we calculated intra-class correlation (ICC) coefficients between each of the DVs and participant and situation conditions. Results show that participant and situation exhibit respective shared variance of $28.5 \%$ and $24.6 \%$ with the expert DV and $28.6 \%$ and $11.7 \%$ with the peer DV. These random effects were therefore included in subsequent models.

To test H1, we estimated a model that included each of the six situational characteristics described above as fixed effects, participant and situation as random effects, and reliance on expert recommendation as the DV. The fit of this model increased significantly over the random-effects-only model (Table $1 ; \chi^{2}=$ $110.41, \mathrm{p}<.001)$, indicating additional explained variance attributable to the situational characteristics. The conditional and marginal $\mathrm{R}^{2}$ for the model were 0.528 and 0.053 , indicating that $52.8 \%$ of the variance in the expert DV was explained by the full model and $5.3 \%$ was explained by the fixed effects (situational characteristics) alone. Results from this model, shown in Table 2, show that reliance on expert recommendation was positively associated with situational characteristics of processing, stress, and task, as predicted by H1. Contrary to our hypothesis, threat was not significantly related to reliance on expert recommendations. These results support the majority of the relationships posited by $\mathrm{H} 1$.

Table 1. Model fit for expert

\begin{tabular}{|c|c|c|c|}
\hline Statistic & $\begin{array}{c}\text { Baseline } \\
\text { Model }\end{array}$ & $\begin{array}{l}\text { Random } \\
\text { Effects } \\
\text { Model }\end{array}$ & Full Model \\
\hline $\mathrm{AIC}$ & 8736.6 & 7268.0 & 7169.5 \\
\hline $\mathrm{BIC}$ & 8748.2 & 7291.0 & 7227.2 \\
\hline \multicolumn{4}{|c|}{ Comparison to previous model } \\
\hline$\chi^{2}$ & - & 1472.7 & 110.41 \\
\hline $\mathrm{p}$ & - & $<0.001$ & $<0.001$ \\
\hline
\end{tabular}

Table 2. Estimates and standard error for expert recommendation model

\begin{tabular}{|l|c|c|}
\hline Dimension & Estimate & Standard Error \\
\hline Intercept) & $1.967^{* * *}$ & 0.210 \\
\hline Processing & $0.113^{* * *}$ & 0.024 \\
\hline Threatening & 0.029 & 0.028 \\
\hline Stressful & $0.110^{* * *}$ & 0.023 \\
\hline Task & $0.090^{* * *}$ & 0.019 \\
\hline Fun & -0.028 & 0.024 \\
\hline Mundane & -0.015 & 0.019 \\
\hline${ }^{* * *} \mathrm{p}<.001$ & \multicolumn{2}{|l}{}
\end{tabular}

To test $\mathrm{H} 2$, we estimated a full model with reliance on peer recommendation as the DV and the same fixed and random effects as for H1. The fit of this model again increased significantly over the randomeffects-only model for peer recommendation (Table 3; $\chi 2=21.73, \mathrm{p}=.0014)$. The conditional and marginal $\mathrm{R}^{2}$ for the model were 0.396 and 0.015 , indicating that $39.6 \%$ of the variance in the peer DV was explained by the full model and $1.5 \%$ was explained by the fixed effects (situational characteristics) alone. Results from this model, shown in Table 4, show that reliance on peer recommendation was positively associated with the fun situational characteristic as posited in $\mathrm{H} 2$; however, contrary to our hypothesis, the coefficient for mundane was not significant. These results provide partial support for $\mathrm{H} 2$.

Table 3. Model fit for peer

\begin{tabular}{|c|c|c|c|}
\hline \multicolumn{5}{|c|}{$\begin{array}{c}\text { Baseline } \\
\text { Model }\end{array}$} & $\begin{array}{c}\text { Random } \\
\text { Effects } \\
\text { Model }\end{array}$ & Full Model \\
\hline AIC & 8223.9 & 7318.1 & 7308.4 \\
\hline BIC & 8235.4 & 7341.2 & 7366.0 \\
\hline \multicolumn{4}{|c|}{} \\
\hline \multicolumn{4}{|c|}{ Comparison to previous model } \\
\hline$\chi 2$ & - & 909.8 & 21.713 \\
\hline$p$ & - & $<0.001$ & .0014 \\
\hline
\end{tabular}


Table 4. Estimates and standard error for peer rating model

\begin{tabular}{|l|c|c|}
\hline Dimension & Estimate & Standard Error \\
\hline Intercept) & $3.147^{* * *}$ & 0.182 \\
\hline Processing & -0.021 & 0.025 \\
\hline Threatening & -0.051 & 0.029 \\
\hline Stressful & 0.001 & 0.024 \\
\hline Task & -0.022 & 0.020 \\
\hline Fun & $0.091^{* * *}$ & 0.025 \\
\hline Mundane & 0.026 & 0.019 \\
\hline${ }^{* * *} \mathrm{p}<.001$ & &
\end{tabular}

\section{Discussion}

Contextual cues reflecting the opinions of both expert and lay users have helped to make online forums a rich and popular platform for knowledge exchange, yet relatively little is understood about how knowledge seekers use these cues to filter and evaluate solutions. This study addresses a central component of this broader research question; namely, how situational factors influence a person's propensity to rely on opinions of experts vs. those of peers. This study breaks new ground in this domain by, to our knowledge, being the first to establish a connection between situational characteristics theory and reliance on different contextual cue sources commonly found on ENP forums. The pattern of results we observed confirms an observable effect of situational characteristics and carries important implications for both theory and practice.

First, as hypothesized by H1, our results show that people are more likely to rely on expert recommendations in situations that are stressful, focused on the attainment of a task, or that require greater amounts of cognitive processing. Although these relationships have not previously been explored in the literature on ENP forums, this result largely accords with consumer product and service literature that has shown a greater reliance on expert opinions in situations where the stakes are utilitarian in nature and/or evaluation of the product or service is beyond the immediate capacity of the consumer [21]. Interestingly, unlike stress, where the locus of the stressor is internally focused, the effect of the threat situational characteristic (an externally-focused stressor) did not have a discernible effect on people's desire to seek out expert opinions. As noted above, a primary distinguishing feature of the threat characteristic is that it does not necessarily imply a negative affective response- a person who feels capable of responding to the external circumstance may view a "threatening" situation as simply a challenge to overcome. Stress, on the other hand, denotes a situation that evokes unpleasant feelings arising not only from a perceived negative circumstance but an inability to adequately deal with the circumstance. This distinction evokes a key tenet coping theory [33], [34], which suggests that people evaluate situations via both a primary appraisal, in which they assess the potential of the situation to promote or harm their well-being, and a secondary appraisal, in which they evaluate their resources and options for coping. Both theory and empirical results suggest that a person's secondary appraisal determines coping strategies [35], and that stress results when secondary appraisal reveals a shortfall of coping resources [36]. Thus, aligning threat and stress situational characteristics with primary and secondary appraisal, respectively, our results imply that reliance on expert opinions on forums has more to do with the person's perceived lack of resources to handle the situation (secondary appraisal) than with the it's perceived menace to well-being (primary appraisal) alone.

With regard to reliance on peer opinions, our results for $\mathrm{H} 2$ suggest that people are more likely to seek out the advice of peers when the situation entails an element of fun or hedonic enjoyment. Because such situations typically involve preferential choices (e.g., choosing a restaurant or a movie for a date) as opposed to normative choices (e.g., choosing the best health care plan), people may be less concerned with identifying the "right" answer, and more inclined to trust opinions of peers with whom they identify and share common interests. This result is largely consistent with findings of consumer product and service literature, which has demonstrated a greater reliance on peer opinions for preference-based or hedonic products or services [37], [38]. Interestingly, the mundane situational characteristic did not seem to induce a greater reliance on peer recommendations as hypothesized. One straightforward explanation for this result might be that people simply might not feel compelled to seek anyone's opinion - neither those of peers nor experts in situations that involve everyday circumstances of relatively little import. For theory, this suggests an interesting boundary condition under which forum contextual cues from either peers or experts may add little marginal value.

In general, our results shed important theoretical light on the ongoing study of ENP forums by showing that situational characteristics do influence the degree to which forum users rely on peer vs. expert opinions. Applying a situational characteristics lens can 
help to explain the apparently discrepant results of previous studies, some of which have shown a preference for peer-based cues [7], [39], and others of which have favored expert-based cues [11]. The situational characteristics we examined might also be fruitfully applied to knowledge evaluation processes using other types of technology platforms. However, it is also interesting to note that the explanatory effect of the situational characteristics, though statistically significant, was somewhat modest compared to the random effects of participant and situation included in the models, as evidenced by the substantial differences in their conditional (full-model) and marginal (fixedeffects-only) $\mathrm{R}^{2}$ values. For theory, this suggests that while situational characteristics are important, individual preferences for peer- vs. expert-based recommendations also appear to be highly salient and should not be ignored in future theoretical development. For example, individual constructs such as self-efficacy [40] and social identity [41] may help to explain some of the variance in the DVs not captured by situational characteristics in this study.

Our results also offer interesting implications for practice, particularly to those who sponsor and moderate online ENP forums. Our findings suggest that the types of contextual cues most salient to forum users depends on the nature of the situation in question. Forums that deal with topics that tend to be serious, complex, or procedural in nature will wish to place greater emphasis on expert-based cues and increase the visibility of these cues to users. On the other hand, users of forums centered on topics that are of a hedonic or preference-based nature will likely appreciate the availability of peer-based cues to guide decisionmaking. Understanding the relevance of these types of cues in different situations can help forum moderators structure their forum and solicit participation from the types of reviewers whose recommendations will be most influential. Currently, many forums present the same user interface, including positioning of contextual cues, for all topics. This research suggests that forum designers might consider differentially weighting/presenting contextual cues based on the topic at hand. A dynamic, more contextually rich interface may allow a better, more meaningful experience.

\section{Limitations \& Future Research}

As with any study, the results of this work should be considered in context of its inherent limitations. First, although the situational characteristics included in this study are strongly grounded in theory and empirical work, they do not necessarily represent an exhaustive set. Future work could expand on these characteristics or examine more detailed subdimensions. Second, our survey was limited to 20 situations that, while taken from literature suggesting likely differences in peer vs. expert opinions [42], may have excluded certain types of ENP forum topics. Finally, though it is arguably a reliable platform for conducting research studies [43], our participants were limited to MTurk workers. Future work might consider soliciting the opinions of other populations of ENP forum users to confirm the generalizability of our results.

\section{Conclusion}

As online forums sponsored by ENP forums continue to grow in popularity, so too does the theoretical and practical need to better understand how these platforms facilitate information exchange. In particular, the availability of contextual cues representing feedback from both expert and lay users offers a rich and useful source of information for forum users, but research surrounding how these cues are actually used by knowledge seekers is scant and results are fragmented and inconsistent. By adopting a situational characteristics perspective, this study lays important theoretical groundwork for future research in this area. Specifically, we show that the propensity to rely on peer vs. expert-based cues depends in part on the nature of the situation in question, though other individual trait-based factors also appear to have influence. We invite further research to build upon our conceptualization of situational characteristics and to explore their explanatory nature in assessing users' decision-making processes on ENP forums.

\section{References}

[1] B. Van Der Heide and Y. S. Lim, "On the Conditional Cueing of Credibility Heuristics: The Case of Online Influence," Communic. Res., vol. 43, no. 5, pp. 672-693, Jul. 2016, doi: $10.1177 / 0093650214565915$.

[2] R. Belk, "You are what you can access: Sharing and collaborative consumption online," J. Bus. Res., vol. 67, no. 8, pp. 1595-1600, Aug. 2014, doi: 10.1016/j.jbusres.2013.10.001.

[3] M. M. L. Wasko, R. Teigland, and S. Faraj, "The provision of online public goods: Examining social structure in an electronic network of practice," Decis. Support Syst., vol. 47, no. 3, pp. 254-265, Jun. 2009, doi: 10.1016/j.dss.2009.02.012.

[4] W. Wong, "Study Shows Americans Prefer Online Forums Over Mainstream Social Media - Business 2 Community," 07-Feb-2019. [Online]. Available: 
https://www.business2 community.com/socialmedia/study-shows-americans-prefer-online-forumsover-mainstream-social-media-02167545.

[Accessed: 03-Jun-2020].

[5] Y. F. Chen, "Herd behavior in purchasing books online," Comput. Human Behav., vol. 24, no. 5, pp. 1977-1992, Sep. 2008, doi:

10.1016/j.chb.2007.08.004.

[6] A. J. Flanagin and M. J. Metzger, "Trusting expert versus user-generated ratings online: The role of information volume, valence, and consumer characteristics," Comput. Human Behav., vol. 29, no. 4, pp. 1626-1634, 2013, doi: 10.1016/j.chb.2013.02.001.

[7] Z. Zhang, Q. Ye, R. Law, and Y. Li, "The impact of e-word-of-mouth on the online popularity of restaurants: A comparison of consumer reviews and editor reviews," Int. J. Hosp. Manag., vol. 29, no. 4, pp. 694-700, 2010, doi:

10.1016/j.ijhm.2010.02.002.

[8] T. Meservy, K. Fadel, C. B. Kirwan, and R. Meservy, "An fMRI Exploration of Information Processing in Electronic Networks of Practice," Manag. Inf. Syst. Q., vol. 43, no. 3, Sep. 2019.

[9] K. Fadel, T. Meservy, and B. Kirwan, "Expectation Disconfirmation in ENP Information Filtering: An fMRI Experiment," AMCIS 2018 Proc., Aug. 2018.

[10] K. J. Fadel, T. O. Meservy, and M. L. Jensen, "Exploring Knowledge Filtering Processes in Electronic Networks of Practice," J. Manag. Inf. Syst., vol. 31, no. 4, pp. 158-181, Jan. 2015, doi: 10.1080/07421222.2014.1001262.

[11] A. Chakravarty, Y. Liu, and T. Mazumdar, "The Differential Effects of Online Word-of-Mouth and Critics' Reviews on Pre-release Movie Evaluation," J. Interact. Mark., vol. 24, no. 3, pp. 185-197, 2010, doi: 10.1016/j.intmar.2010.04.001.

[12] "Situation | Definition of Situation by Oxford Dictionary on Lexico.com also meaning of Situation." [Online]. Available:

https://www.lexico.com/en/definition/situation. [Accessed: 03-Jun-2020].

[13] R. Meyer, "Taxonomy of situations and their measurement," Oxford Handbooks Online, no. February, pp. 1-24, 2015, doi: 10.1093/oxfordhb/9780199935291.013.22.

[14] Y. Yang, S. J. Read, and L. C. Miller, "The Concept of Situations," Soc. Personal. Psychol. Compass, vol. 3, no. 6, pp. 1018-1037, Dec. 2009, doi: 10.1111/j.1751-9004.2009.00236.x.

[15] J. F. Rauthmann, R. A. Sherman, and D. C. Funder, "Principles of Situation Research: Towards a Better Understanding of Psychological Situations," Eur. J. Pers., vol. 29, no. 3, pp. 363-381, May 2015, doi: 10.1002/per.1994.

[16] M. A. Ten Berge and B. De Raad, "Taxonomies of situations from a trait psychological perspective. A review," European Journal of Personality, vol. 13, no. 5. pp. 337-360, 1999, doi: 10.1002/(SICI)10990984(199909/10)13:5<337::AID-

PER363>3.0.CO;2-F.
[17] J. A. Edwards and A. Templeton, "The structure of perceived qualities of situations," European Journal of Social Psychology, vol. 35, no. 6. pp. 705-723, Nov-2005, doi: 10.1002/ejsp.271.

[18] Y. Yang, S. J. Read, and L. C. Miller, "A taxonomy of situations from Chinese idioms," J. Res. Pers., vol. 40, no. 5, pp. 750-778, 2006, doi: 10.1016/j.jrp.2005.09.007.

[19] S. Oreg, J. A. Edwards, and J. F. Rauthmann, "The situation six: Uncovering six basic dimensions of psychological situations from the Hebrew Language," J. Pers. Soc. Psychol., vol. 118, no. 4, pp. 835-863, 2020, doi: 10.1037/pspp0000280.

[20] R. A. Horstmann, K. T., Rauthmann, J. F., \& Sherman, "The measurement of situational influences," SAGE Handb. Personal. Individ. Differ., 2017.

[21] H. T. Keh and J. Sun, "The Differential Effects of Online Peer Review and Expert Review on Service Evaluations: The Roles of Confidence and Information Convergence," J. Serv. Res., vol. 21, no. 4, pp. 474-489, Nov. 2006, doi: 10.1177/1094670518779456.

[22] P. Racherla and W. Friske, "Perceived 'usefulness' of online consumer reviews: An exploratory investigation across three services categories," Electron. Commer. Res. Appl., vol. 11, no. 6, pp. 548-559, 2012, doi: 10.1016/j.elerap.2012.06.003.

[23] H. S. Bansal and P. A. Voyer, "Word-of-Mouth Processes within a Services Purchase Decision Context," J. Serv. Res., vol. 3, no. 2, pp. 166-177, 2000, doi: 10.1177/109467050032005.

[24] A. De Bruyn and G. L. Lilien, "A multi-stage model of word-of-mouth influence through viral marketing," Int. J. Res. Mark., vol. 25, no. 3, pp. 151-163, 2008, doi: 10.1016/j.ijresmar.2008.03.004.

[25] M. Gilens and N. Murakawa, Elite Cues and Political Decision-Making, vol. 6. 2002.

[26] F. D. Vennik, S. A. Adams, M. J. Faber, and K. Putters, "Expert and experiential knowledge in the same place: Patients' experiences with online communities connecting patients and health professionals," Patient Educ. Couns., vol. 95, no. 2, pp. 265-270, May 2014, doi: 10.1016/j.pec.2014.02.003.

[27] A.-M. Kranzbühler, M. H. P. Kleijnen, P. W. J. Verlegh, and M. Teerling, "The Differential Effects of Peer and Expert Ratings on Choice," Mark. Sci. Inst. Work. Pap. Ser., no. 15-117, pp. 1-34, 2015.

[28] D. Smith, S. Menon, and K. Sivakumar, "Online peer and editorial recommendations, trust, and choice in virtual markets," J. Interact. Mark., vol. 19, no. 3, pp. 15-37, Jan. 2005, doi: 10.1002/dir.20041.

[29] Kelman HC., "Compliance, identification, and internalization: Three processes of attitude change," J. Conflict Resolut., vol. 1, pp. 51-60, 1958.

[30] M. J. Metzger, A. J. Flanagin, and R. B. Medders, "Social and heuristic approaches to credibility evaluation online," J. Commun., vol. 60, no. 3, pp. 413-439, Sep. 2010, doi: 10.1111/j.1460- 
2466.2010.01488.x.

[31] Jordan Grimmer, "Experts vs. Friends: The Definitive Guide to Who Influences Us and Why," Medium, 2016. [Online]. Available:

https://medium.com/bestcompany/experts-vsfriends-the-definitive-guide-to-who-influences-usand-why-6a0aa609c8c0. [Accessed: 18-Sep-2019].

[32] M. B. (2015). Bates, D., Maechler, M., Bolker, B., Walker, S., Christensen, R. H. B., Singmann, H., ... \& Bolker, "Package 'lme4,", 2020.

[33] R. S. Lazarus, Psychological stress and the coping process. New York, New York, USA: McGraw-Hill, 1966.

[34] R. S. Lazarus and S. Folkman, Stress, Appraisal, And Coping. New York, 1984.

[35] R. S. Lazarus and S. Folkman, "Transactional theory and research on emotions and coping," Eur. J. Pers., vol. 1, no. 3, pp. 141-169, 1987, doi: $10.1002 /$ per. 2410010304 .

[36] J. Walinga, "Toward a theory of change readiness: The roles of appraisal, focus, and perceived control," J. Appl. Behav. Sci., vol. 44, no. 3, pp. 315-347, Sep. 2008, doi: 10.1177/0021886308318967.

[37] J. H. Huang and Y. F. Chen, "Herding in online product choice," Psychol. Mark., vol. 23, no. 5, pp. 413-428, May 2006, doi: 10.1002/mar.20119.
[38] J. A. Chevalier and D. Mayzlin, "The effect of word of mouth on sales: Online book reviews," Journal of Marketing Research, vol. 43, no. 3. pp. 345-354, Aug-2006, doi: 10.1509/jmkr.43.3.345.

[39] S. Hazari, B. J. Bergiel, and B. N. Sethna, "Hedonic and utilitarian use of user-generated content on online shopping websites," J. Mark. Commun., vol. 23, no. 6, pp. 572-591, 2017, doi: 10.1080/13527266.2016.1143383.

[40] A. Bandura, Self-efficacy: The exercise of control. 1997.

[41] H. Tajfel and J. Turner, "An integrative theory of inter-group conflict," in The social psychology of intergroup relations, 1979.

[42] "Experts vs. Friends: The Definitive Guide to Who Influences Us and Why." [Online]. Available: https://medium.com/bestcompany/experts-vsfriends-the-definitive-guide-to-who-influences-usand-why-6a0aa609c8c0. [Accessed: 01-Feb-2020].

[43] M. Buhrmester, T. Kwang, and S. D. Gosling, "Amazon's Mechanical Turk: A new source of inexpensive, yet high-quality data?," in Methodological issues and strategies in clinical research (4th ed.)., American Psychological Association, 2015, pp. 133-139.

\section{Appendix}

Table 1. Informational Attributes

A PROCESSING based situation requires deeper and effortful cognitive information processing (e.g., filing taxes).To what extent do you agree or disagree that each of the following situations is PROCESSING based?

A THREATENING situation is one where many external threats and obstacles must be overcome (e.g., surgery). To what extent do you agree or disagree that each of the following situations is THREATENING?

A STRESSFUL situation is one where you need to deal with (internal) negative events that may cause distress (e.g., reporting the unethical behavior of a close colleague). To what extent do you agree or disagree that each of the following situations is STRESSFUL?

A FUN situation has elements of pleasantness and enjoyability (e.g., attending a concert). To what extent do you agree or disagree that each of the following situations is FUN?

A TASK based situation is focused on the accomplishment of an important or urgent task (e.g., paying a bill). To what extent do you agree or disagree that each of the following situations is TASK based?

A MUNDANE situation is typically routine and repetitious in nature (e.g., running weekly errands at work). To what extent do you agree or disagree that each of the following situations is MUNDANE? 
Table 2. Hypothetical Situations

\begin{tabular}{|l|}
\hline Selecting my fantasy football lineup \\
\hline Choosing among car insurance providers \\
\hline Deciding on a travel destination \\
\hline Determining whether to do business with a certain company \\
\hline Seeking advice on a diet/exercise plan \\
\hline Trying to make sense of a current news event \\
\hline Deciding whether to lease or purchase a vehicle \\
\hline Deciding which credit card is best for me \\
\hline Deciding who to vote for \\
\hline Seeking relationship advice \\
\hline Determining my romantic capability with someone \\
\hline Deciding on a career \\
\hline Deciding to try a new restaurant \\
\hline Determining whether to purchase a new personal computer or mobile phone \\
\hline Selecting a college major \\
\hline Determining whether to purchase a certain product \\
\hline Seeking advice on how to get a good loan \\
\hline Seeking financial advice \\
\hline Determining whether a movie is worth seeing \\
\hline Seeking advice on purchase a home \\
\hline
\end{tabular}

